\title{
DETAILED MODELLING AND ANALYSIS OF DIGITAL MHO DISTANCE RELAY WITH SINGLE-POLE OPERATION
}

\author{
Paulo Henrique Barbosa de Souza Pinheiro, \\ mayara Helena Moreira Nogueira dos Santos, \\ Angelo Cesar Colombini, Bruno Wanderley França, \\ Marcio Zamboti Fortes*
}

\author{
Fluminense Federal University, Engineering School, Electrical Engineering Department, 24210-240 Niteroi, RJ, \\ Brazil \\ * corresponding author: mzamboti@id.uff.br
}

\begin{abstract}
This paper introduces a methodology for modelling a digital admittance-type distance relay using PSCAD/EMTDC. The proposed distance relay was tested in a simulation of the Brazilian power grid with predetermined fault scenarios. The goal of this paper is to make a detailed evaluation of the mho distance relay. The main aspects include the correct operation of the distance relay, fault resistance effects on the mho characteristics, and the fault detection time of this relay. A new approach to analyse the fault detection time is presented, considering several simulated fault scenarios. The results demonstrate that the fault resistance influences the fault detection time and severely affects the distance relay's general performance. The fault detection time is not constant. It varies within a time interval, considering different fault types, fault locations, and fault resistances. The confidence interval calculation provides a detailed range of the fault detection time, considering its upper and lower limits.
\end{abstract}

KEYWORDs: Electrical engineering, power system protection, PSCAD/EMTDC, time-domain analysis.

\section{INTRODUCTION}

Power system protection is a wide area of study with many challenges regarding electrical equipment safety and power system reliability. A well-designed protection scheme must guarantee these two main aspects and remove any undesired conditions that might happen. For high-voltage (HV) and extra-high-voltage (EHV) systems, transmission line protection brings many significant improvements, mainly when it includes distance relay modelling.

Distance relays protect transmission lines. They respond to the impedance between the relay and the fault location 1. The basic principle of distance protection includes the impedance measurement to the fault location in the transmission line. Instrument transformers provide voltage and current signals at the relay location. Through these signals, it is possible to estimate the fault location and to determine which fault occurred.

The increasing insertion of digital distance relays in electrical power systems led to several works regarding their modelling. The goals of these works are, in general, to make an accurate evaluation of their algorithms and to propose new ones to solve common problems of distance protection.

Some common problems related to the distance protection can be found in [2] 5 . Authors in [2] bring a methodology to model a distance relay using a Real-Time Digital Simulator (RTDS). They highlight every step needed to model the digital distance relay in the following order:

- Signal conditioning unit

- Data acquisition unit

- Data processing unit

Following this sequence, it is possible to model a complete digital relay, beginning with voltage and current measurements and up to the command signals to trip circuit-breakers. This reference considers two different operational characteristics for the distance relay. They are mho and quadrilateral. The most important reported problems are fault detection and fault classification. This reference applies the three-pole operation scheme for the distance relay model. It means that, for every possible fault type in a three-phase system, the relay will open the circuit-breakers belonging to the three phases of the system.

Another problem of great importance in the distance protection is the fault resistance during a short-circuit. It can result in the distance relay carrying out an incorrect operation. An extension of an existing ground distance relay algorithm to include phase distance relays is presented in [3. The algorithm uses a fault estimation process to improve the efficiency of the distance protection. Results show that the fault resistance compensation 
algorithm is suitable for online applications. It is relevant to highlight that conventional distance relays might present some limitations to detect and classify faults with high impedance. One of this paper's contributions includes proving it.

In [4], the authors investigate the effects of new FACTS (Flexible AC Transmission System) installations on existing distance relays. The effect of the presence of a UPFC (Unified Power Flow Controller) was evaluated. It is a controller that can simultaneously support bus voltage and control the flow of power. It is reported that a conventional distance relay will calculate the distance to the fault location based on the bus voltage and current. If this distance is within a protection zone, the relay will clear the fault. When the UPFC is connected to the system, the impedance seen by the distance relay must include the impedance of the device. In addition, the UPFC has a by-pass circuit-breaker to isolate it during fault conditions. Several short circuits were simulated in the power system considered. The main conclusions are, during a steady-state operation, the distance relay will operate correctly. Furthermore, during most of the fault conditions, the by-pass circuit-breaker isolates the device. Then, it will not affect the correct operation of the protection relays. Reactive compensation devices must be considered in the distance protection because they can affect the impedance measurement by the distance relay. Problems related to FACTS devices include overreaching the distance protection [6]. In the presence of faults with impedance, there is also the possibility of underreaching [7].

Beyond the aforementioned problems related to the distance protection, another critical condition that might happen in electrical power systems and can severely affect the distance protection is a power swing. Power swings originate from the rotor angle oscillations from synchronous generators. These power swings can be harmful to distance protection. They might cause impedance trajectory encroachment on the relay protection zones [5].

Unlike electrical networks with power generation mostly using synchronous generators, distance protection using conventional mho and quadrilateral characteristics is affected by renewable energy sources connected to the transmission network using electronic power converters. The adaptive mho characteristic presented in 8 is suitable for solving problems of this type. The topology changes in the electrical systems highlight the need to study the existing protection algorithms and identify their limitations. Then, it is possible to propose new solutions that suit the new scenarios.

There are many types of distance relays, from electromechanical ones to the latest computer relays. Conventional types of distance relays found in the literature (e.g., [1] and [9]) are:

- Impedance-type distance relay

- Modified type distance relay

- Reactance type distance relay

- Admittance type distance relay

- Quadrilateral type distance relay

The last characteristic can be found only in solid-state or computer relays, while the others exist in both the electromechanical and the digital relays. The two commonly applied types of distance protection elements include admittance type and quadrilateral type distance relays [10].

The paper aims to describe the process of modelling a digital admittance distance relay (mho) and to make a detailed evaluation of its behaviour during several simulated fault scenarios. This evaluation focuses mainly on its correct operation, analyses the effects of fault impedances on distance protection, and the fault detection time of the proposed distance relay. A novel approach to fault detection time is presented. It consists of the confidence interval calculation to provide a range of plausible values of fault detection time, including its upper and lower limits. The distance relay modelling and its evaluation were conducted using the program PSCAD/EMTDC (Power System Computer-Aided Design/Electromagnetic Transients including Direct Current). The simulated transmission line belonging to the Brazilian power grid is used to evaluate the distance relay's performance.

The paper is structured as follows: section 2 presents related works regarding the modelling of distance relays, section 3 depicts the proposed distance relay, section 4 provides more details about the studied scenario and presents simulation results, section 5 presents the conclusions.

\section{RELATED WORKS}

This section presents related works about the modelling of digital distance relays. Some of them are used as guides to the development of the proposed model and to the evaluation of some parameters concerning distance protection.

An mho distance relay is proposed in [11, using the EMTPWorks package. This implementation is suitable, considering the nonlinear nature of an arcing fault, which requires a time-domain implementation for the distance relay. Hence, it is possible to determine if the fault will or will not be detected. The authors modelled the 
distance relay only for ground faults. Due to this, it is not possible to evaluate phase faults. The current distance relay presented in this paper also implements the mho characteristic. Furthermore, it includes the possibility to test all fault types in its algorithm. Several simulated faults scenarios are considered to check the algorithm's general performance. These fault scenarios also allow the verifying of limitations of the distance relay using the transient analysis program PSCAD/EMTDC.

A quadrilateral characteristic of distance protection is presented and tested in [12. The performance of this quadrilateral distance relay is evaluated using the model of an electrical power system located in India. Ground and phase faults are simulated, varying many different conditions in the power system. These conditions are fault resistance, power transfer angle, and line charging. The proposed distance relay uses the following sequence in its structure:

- Anti-aliasing filter

- Fast Fourier transform

- Sequence filter

- Impedance calculation

- Fault detection and classification

- Trip signal

Following this sequence, it is possible to extract the fundamental components of voltage and current signals during a fault. These signals allow to make an accurate estimation of the impedance to the fault location and correctly send the trip signal to the circuit-breakers. It is possible to notice, in this reference, that the quadrilateral characteristic is suitable to cover faults with a high impedance, and varying the angle of power transfer affects the impedance calculation. Some of these steps adopted in 12 are applied to the mho distance relay model proposed in this paper (e.g., the anti-aliasing filter and the fast Fourier transform). Further details are given in section 3 .

An approach to distance protection using the S-transform is presented in [13. It is an invertible timefrequency localization technique that combines elements of the wavelet transform and the short-time Fourier transform. The S-transform was applied to the voltage and current signals to generate the S-matrix. The changing of the energy applies to the process of fault detection and the estimated phasors are used for the impedance calculation. Applying this technique allows the possibility to detect the faults within less than a half-cycle of the fundamental frequency. The total time, of detecting the fault and sending the trip signal, for the proposed scheme, is about 20 samples (one cycle based on the fundamental frequency of $50 \mathrm{~Hz}$ ). In this paper, the confidence interval calculation of the fault detection time is proposed as an alternative method to treat this parameter. Different fault scenarios influence the fault detection time. With this methodology, it is possible to obtain an accurate range of these values, considering best and worst scenarios. These scenarios were elaborated considering different fault resistances, different fault locations, and different fault types. The last parameter evaluated in this paper is the correct operation of the distance relay facing these different scenarios.

After this review of related works about distance relays, modelling, section 3 will depict the process of modelling the proposed mho distance relay.

\section{Digital mho Distance Relay}

In this section, the algorithm for the implemented mho distance relay using the program PSCAD/EMTDC is presented. This transient analysis software is used by engineers, researchers, and students from utilities, manufactures, consultants, research and academic institutes 14.

The digital distance relay modelled in this paper uses the admittance characteristic in its protection zones. It is also known as the mho distance relay. The admittance characteristic is a circular tripping characteristic. It can pass through the origin on the $\mathrm{R}$-X diagram or might be offset from it [15]. The mho distance relay with its circular characteristic passing through the origin is the self-polarized mho distance relay. This is the characteristic applied in this paper. The self-polarized mho distance relay decides to trip the circuit-breakers if the measured impedance is located within its protection zone. This study case considered two protection zones, zone 1 and zone 2 .

Protection zone 1 has an instantaneous action characteristic and underreaches to the remote bus of the transmission line. However, protection zone 2 has a time delay before sending the trip signal to the circuitbreakers of the protected line, and it overreaches to the remote bus. This time delay is used for protection coordination. In general, it is also used in a protection zone 3. This third zone has to be set such as to protect the longest adjacent line and to protect $20 \%$ beyond that line to provide a backup to the remote circuit-breakers [16]. This paper did not consider external faults, therefore, the third zone was not implemented in this study. 
The first step of the distance relay modelling is to acquire voltage and current signals from instrument transformers. These analog signals must be applied to anti-aliasing filters to limit the noise effects and unwanted components of higher-frequencies over the sampled data [17]. These higher frequencies can emerge mainly due to traveling waves during transient conditions in the transmission line.

The anti-aliasing filter can be understood as a prefiltering stage. It is used, in general, if the Nyquist frequency of the input is too high or if the signal is not bandlimited. Even if the input signal is naturally bandlimited, wideband noise may exist in the higher frequencies range. During the sampling of this input signal, these noise components would be aliased into the low-frequency band. To avoid this phenomenon, the input signal is forced to be bandlimited to frequencies below one half of the desired sampling rate using this low pass filter (i.e., anti-aliasing filter), [18] presents more details about the subject. In this paper, the function shown in 1 simulates an analog, low pass, anti-aliasing filter consisting of a state variable formulation with trapezoidal integration [19].

$$
Y(t)=L^{-1}\left\{\frac{X(s)}{A_{0}+A_{1}\left(\frac{s}{\omega}\right)+A_{2}\left(\frac{s}{\omega}\right)^{2}+A_{3}\left(\frac{s}{\omega}\right)^{3}+A_{4}\left(\frac{s}{\omega}\right)^{4}+A_{5}\left(\frac{s}{\omega}\right)^{5}+A_{6}\left(\frac{s}{\omega}\right)^{6}}\right\}
$$

where:

$s$ - Laplace variable

$\omega=2 \cdot \pi \cdot F G(\mathrm{rad} / \mathrm{s})$

$X(s)$ - input in the Laplace domain

$L^{-1}$ represents inverse Laplace transform

The values of the coefficients in 1 are $F G=0.421875 \times$ sampling frequency; $A_{0}=1.0 ; A_{1}=3.8637$; $A_{2}=7.464 ; A_{3}=9.1415 ; A_{4}=7.464 ; A_{5}=3.8637 ; A_{6}=1.0$. After the anti-aliasing filter stage, the input signals are sampled at a frequency greater than double the highest harmonic frequency of interest according to the Nyquist criterion 20. Therefore, in this case, as the distance relay is interested only in the fundamental frequency, the $7^{\text {th }}$ harmonic is chosen as the highest component of interest. The data sampling frequency is 16 samples/cycle. Then, these data are written into a buffer, and the harmonic computations are based on the fast Fourier transform (FFT) technique [21]. The FFT computation is detailed in 2 [22, 23]. The FFT results are in the format given by 3 the magnitude computation uses 4 , and the phase angle computation uses 5 .

$$
X(k)=\sum_{n=0}^{N / 2-1} x(2 n) W_{N / 2}^{n k}+W_{N}^{k} \sum_{n=0}^{N / 2-1} x(2 n+1) W_{N / 2}^{n k}
$$

where:

$$
\begin{aligned}
& X(k) \text { - output signal vector } \\
& x(n) \text { - input signal vector decomposed into } x(2 n) \text { and } x(2 n+1) \\
& N-\text { number of samples of the input signal } \\
& k-\text { harmonic frequency index } \\
& W_{N}=e^{-j 2 \pi / N} \\
& \qquad X(k)=\operatorname{Real}[X(k)]+j \operatorname{Imag}[X(k)] \\
& \operatorname{Mag}(k)=\sqrt{\operatorname{Real}[X(k)]^{2}+\operatorname{Imag}[X(k)]^{2}} \\
& \operatorname{Phase}(k)=\tan ^{-1}\left(\frac{\operatorname{Imag}[X(k)]}{\operatorname{Real}[X(k)]}\right)
\end{aligned}
$$

where:

$\operatorname{Real}[X(k)]$ - real portion of $X(k)$ 


$$
\operatorname{Imag}[X(k)] \text { - imaginary portion of } X(k)
$$

These steps of anti-aliasing filtering, data sampling, harmonic, and phase angle computations, depicted in Figure 1 are used to estimate the fundamental phasors of voltage and current signals. They are used to calculate the impedance of the fault loops in the relay. After this step of phasors estimation, the impedances are calculated, for both phase and ground elements, covering all types of faults that might happen in a three-phase system. They are the following:

- Phase-ground faults

- Phase-phase faults

- Phase-phase-ground faults

- Three-phase faults

- Three-phase-ground faults

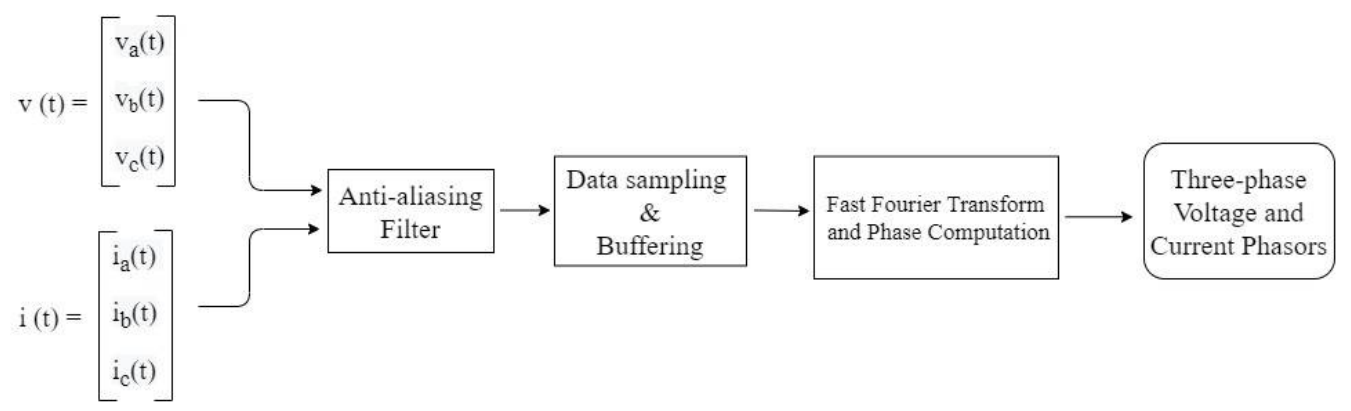

Figure 1. Signal conditioning unit.

The impedance computation of phase elements uses 6 , and the impedance of ground elements uses 7 24.

$$
Z_{1}=\frac{V_{\phi 1}-V_{\phi 2}}{I_{\phi 1}-I_{\phi 2}}(\Omega)
$$

where:

$Z_{1}$ - positive-sequence impedance $(\Omega)$

$V_{\phi 1}$ - phase 1 voltage $(\mathrm{V})$

$V_{\phi 2}$ - phase 2 voltage $(\mathrm{V})$

$I_{\phi 1}$ - phase 1 current $(\mathrm{A})$

$I_{\phi 2}$ - phase 2 current $(\mathrm{A})$

$$
Z_{1}=\frac{V_{\phi}}{I_{\phi}-k I_{0}}(\Omega)
$$

where:

$V_{\phi}$ - phase voltage $(\mathrm{V})$

$I_{\phi}$ - phase current (A)

$k$ - zero-sequence compensation factor

$I_{0}$ - zero-sequence current $(\mathrm{A})$

As it is possible to notice in 7 there are two additional variables. The zero-sequence compensation factor $k$, and the zero-sequence current $I_{0}$. Their computation uses 8 and 9 , respectively.

$$
k=\frac{Z_{0}-Z_{1}}{Z_{1}}
$$

where: 
$Z_{0}$ - zero-sequence impedance of the protected line $(\Omega)$

$Z_{1}$ - positive-sequence impedance of the protected line $(\Omega)$

$$
I_{0}=\frac{1}{3}\left(I_{a}+I_{b}+I_{c}\right)(A)
$$

where:

$$
I_{a}, I_{b}, I_{c} \text { - phase currents (A) }
$$

The zero- and positive-sequence impedances used to calculate $k$ in 8 will be given in section $4 Z_{1}$ being the positive-sequence impedance, a complex number following the format given in 10 , the trip decision of the mho distance relay has the following conditions [25, 26]: First, it calculates the difference between the vectors $Z_{\text {set }}$ and $Z_{1}$, denoted by 11 , and then it evaluates if the angle between $d Z$ and $Z_{1}$ is within a specific range, the flowchart of the trip decision is depicted in Figure 2.

$$
Z_{1}=R+j X(\Omega)
$$

where:

$R$ - positive-sequence resistance $(\Omega)$

$X$ - positive-sequence reactance $(\Omega)$

$$
d Z=Z_{\text {set }}-Z_{1}(\Omega)
$$

where:

$Z_{\text {set }}$ - protection zone's reach setting $(\Omega)$

Table 1 depicts the reach settings for protection zone 1 and protection zone 2 used in this study case. These settings are based on the positive-sequence impedance of the transmission line model considered in this paper. The reach settings of zone 1 and zone 2 are $80 \%$ and $120 \%$ of its positive-sequence impedance, respectively.

\begin{tabular}{lc}
\hline Protection zone & Settings \\
\hline Zone 1 & $35.644 \angle 86.54^{\circ} \Omega$ \\
Zone 2 & $53.46 \angle 86.54^{\circ} \Omega$ \\
\hline
\end{tabular}

TABLE 1. Reach settings.

The reach settings denoted in Table 1 are in secondary ohms, because of the turns ratio from instrument transformers. Section 4 presents more details about them.

The distance relay must correctly measure the impedance of the phase and ground elements, which is done using 6 and 7 Through the conditions about the trip decision, the mho distance relay must correctly classify the faulted phases. Then, it will send the trip signal only to the respective circuit-breakers. Figure 3 shows the logical scheme adopted to trip only the circuit-breakers of the faulted phases.

The logical scheme detailed in Figure 3 can be understood as follows, if the fault is within the protection zone 1 or 2 , the relay will detect which element, phase or ground, is in logical level 1 and send the trip signal to the respective circuit-breakers. As it is possible to notice, the phase elements are $\mathrm{AB}, \mathrm{AC}, \mathrm{BC}$ and the ground elements are $\mathrm{AG}, \mathrm{BG}$, and $\mathrm{CG}$. The protection zone 2 has a time delay component, configured, in this case, with a delay of 20 cycles of the fundamental frequency, used for protection coordination. The variable $B R K_{X}$ denotes the circuit-breaker's status (e.g., opened or closed), in this case, used to hold its current position.

A consideration of this distance relay model is another time delay considered to open the circuit-breakers. Circuit-breakers are electromechanical devices, so they do not open instantaneously. Analysing real fault oscillograms, it is possible to conclude that the mean time interval between the trip signal and the effective opening of the circuit-breaker is $33 \mathrm{~ms}$. These real oscillograms belong to the power line studied in this paper (i. e., Serra da Mesa - Samambaia C3), located in the Brazilian power grid. Simulating this time delay allows more accurate results.

The last thing to consider about the distance relay model is to plot the protection zones and the impedance trajectory from phase and ground elements, so it is possible to verify possible unwanted encroachment of measured 


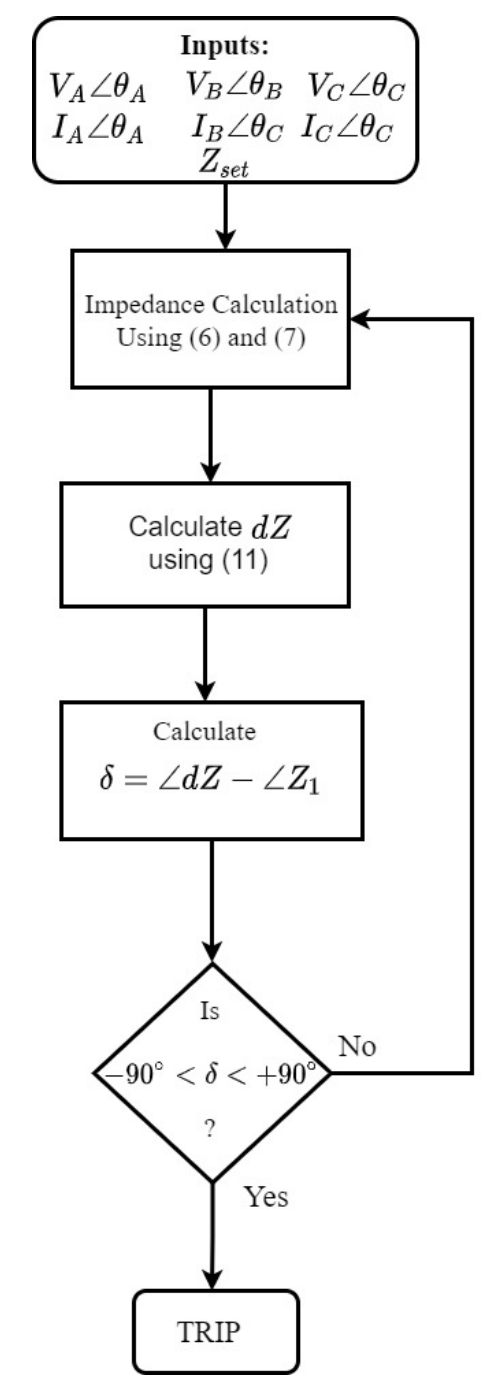

Figure 2. Flowchart of trip decision.

\begin{tabular}{lccc}
\hline \multicolumn{4}{c}{ Length $0-12.474 \mathrm{~km}$ and $234.732-249 \mathrm{~km}$} \\
\hline & Conductor & Ground wire 1 & Ground wire 2 \\
Name & Rail & Dotterel & OPGW \\
Inner radius $(\mathrm{m})$ & 0.003705 & - & - \\
Outer radius $(\mathrm{m})$ & 0.014805 & 0.0077 & 0.0077 \\
DC resistance $(\Omega / \mathrm{km})$ & 0.0599 & 0.324 & 0.429 \\
Bundled sub-conductors & 4 & - & - \\
\hline & Length $12.474-234.732 \mathrm{~km}$ & \\
\hline & Conductor & Ground wire 1 & Ground wire 2 \\
Name & Rail & $3 / 8 ”$ EHS & OPGW \\
Inner radius $(\mathrm{m})$ & 0.003705 & - & - \\
Outer radius $(\mathrm{m})$ & 0.014805 & 0.004572 & 0.0062 \\
DC resistance $(\Omega / \mathrm{km})$ & 0.0599 & 4.1994 & 0.735 \\
Bundled sub-conductors & 4 & - & - \\
\hline
\end{tabular}

TABle 2. Conductors and ground wire data. 
Zone 1
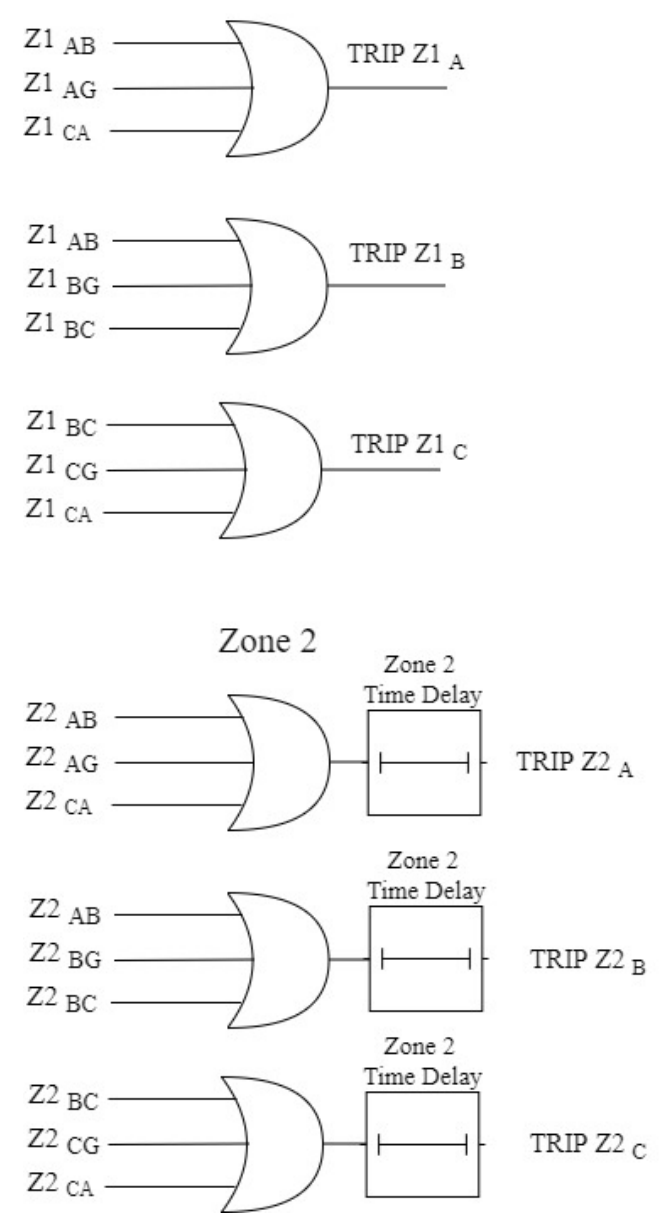

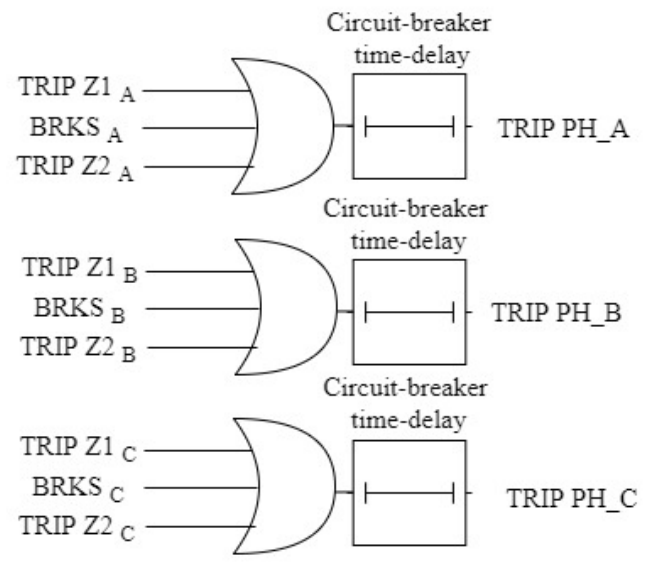

Figure 3. Logical scheme to trip circuit-breakers.

impedances in the protection zones and to check incorrect operation conditions. The circular characteristic of the self-polarized mho distance relay passes through the origin on the $\mathrm{R}-\mathrm{X}$ diagram. It is necessary to generate this offset condition as follows: first, one must calculate one point on the $\mathrm{R}$ axis and another on the $\mathrm{X}$ axis using 12 and 13 respectively.

$$
\begin{aligned}
& R_{\text {axis }}=\text { radius } \times \cos \left(\theta_{\text {line }}\right) \\
& X_{\text {axis }}=\text { radius } \times \sin \left(\theta_{\text {line }}\right)
\end{aligned}
$$

where:

$R_{\text {axis }}$ - offset on the real axis $(\mathrm{R})(\Omega)$

$X_{\text {axis }}$ - offset on the imaginary axis $(\mathrm{X})(\Omega)$

radius - one half of the impedance magnitude of the protection zone $(\Omega)$

$\theta_{\text {line }}$ - angle of the transmission line $\left(^{\circ}\right)$

Table 1 gives the magnitude and angle values of both protection zones 1 and 2. With the magnitude values of these protection zones, it is possible to plot the circular characteristic concerning each zone. After this step, two different signals must be calculated, one for the $\mathrm{R}$ axis and one for the $\mathrm{X}$ axis. They must be plotted as one in function of the other. These signals are calculated in the time domain for R and X using 14 and 15 respectively.

$$
R_{\text {signal }}=\text { radius } \times \cos (\omega t)+R_{\text {axis }}
$$




$$
X_{\text {signal }}=\text { radius } \times \sin (\omega t)+X_{\text {axis }}
$$

where:

$R_{\text {signal }}$ - signal generated for the real axis

$X_{\text {signal }}$ - signal generated for the imaginary axis

$\omega$ - angular frequency ( $\mathrm{rad} / \mathrm{s})$

$t$ - simulation runtime $(\mathrm{s})$

In this study case, the angular frequency $\omega$ was empirically chosen as $360 \pi \mathrm{rad} / \mathrm{s}$. The simulation runtime increases with a time step $\Delta \mathrm{t}=10 \mu \mathrm{s}$. Calculating $R_{\text {signal }}$ and $X_{\text {signal }}$ for the protection zones 1 and 2 . Plotting them as $\left(R_{\text {signal }}, X_{\text {signal }}\right)$ results in the characteristic shown in Figure 4 . After this description of how to model the mho distance relay, section 4 presents the details about the studied scenario and the results.

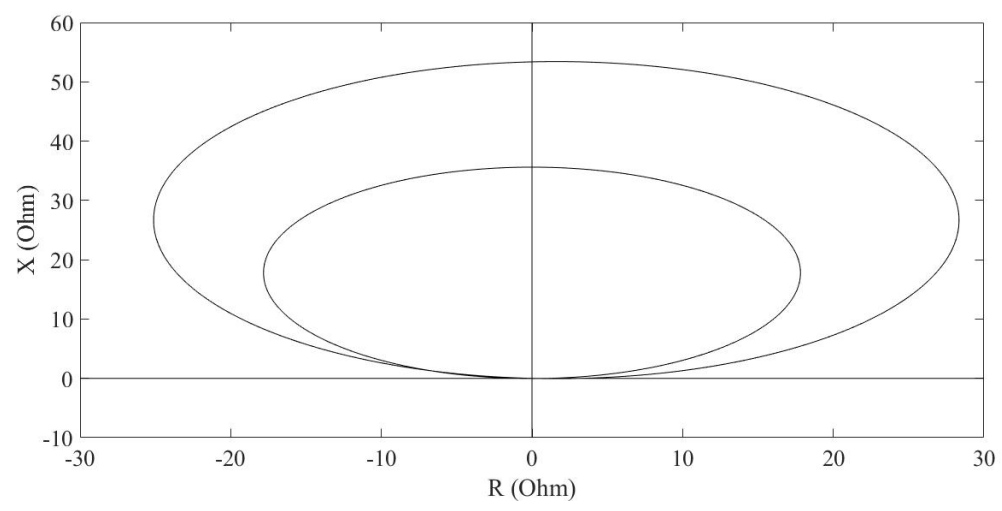

FiguRE 4. Mho circular characteristic.

\section{Simulation AND RESUlts}

To test the distance relay presented in section 3 , the model of a transmission line belonging to the Brazilian power grid is used. The transmission line of interest interconnects the substations of Serra da Mesa and Samambaia. This line is part of the Northern-Southern interconnection of the Brazilian transmission power grid. The transmission line of interest uses the Frequency-Dependent (Phase) Model available in PSCAD/EMTDC. This is the most accurate transmission line model available. [27] and [28] presents more details about this frequency-dependent model.

The model of the transmission line Serra da Mesa - Samambaia C3 uses the geometric characteristic of its predominant tower, as shown in Figure 5. The line is $500 \mathrm{kV}$ voltage rated, and it has a series capacitor for reactive compensation at the Samambaia substation. Its length is $249 \mathrm{~km}$ and it employs a complete transposition cycle, as shown in Figure 6. Conductors and ground wire data are described in Table 2.

For this paper, the series capacitor located at the Samambaia substation will be with its by-pass circuit-breaker closed, so the distance protection will not be under the effects of reactive compensation. The distance relay tests are performed with several fault scenarios applied to the transmission line Serra da Mesa - Samambaia C3. The following parameters are changed to create different scenarios: fault types, fault locations, and fault resistances. With these parameters, the proposed distance relay is accurately evaluated for its general performance. These parameters were changed using the multiple run algorithm available in PSCAD/EMTDC. The algorithm applies 11 different fault types in 12 different locations along the transmission line. In addition, with 5 different values of fault resistance. The fault types are detailed in Table 3 Figure 7 depicts the transmission line single-line diagram, highlighting the relay and instrument transformers' location and the distances considered to the simulated faults. The fault resistance values are $0,1,10,50$ and $100 \Omega$.

This paper aims to evaluate the performance of a digital mho distance relay. The main aspects are the correct operation of the relay in isolating the various types of simulated faults. It means that the relay must trip the circuit-breakers belonging to the faulted phase and the healthy phases must remain live. A comparison of the influence of fault resistance in the correct operation of this distance relay is presented. The last evaluation is the confidence interval calculation of the fault detection time.

The positive-sequence and zero-sequence impedances of the transmission line are obtained using the "line constants" auxiliary routine available in PSCAD/EMTDC. These impedance values are applied to the distance 


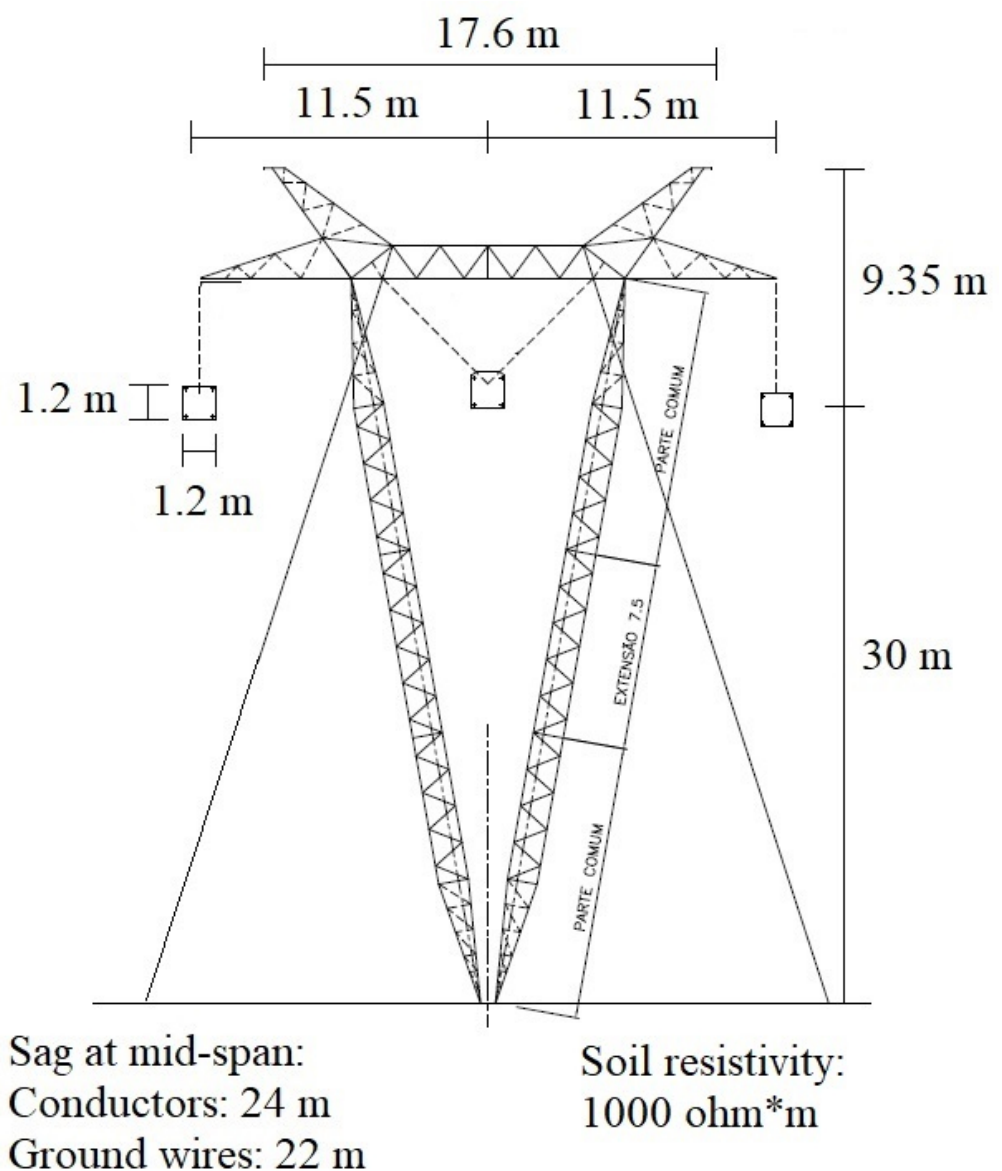

FIGURE 5. Geometric characteristic of the predominant tower.

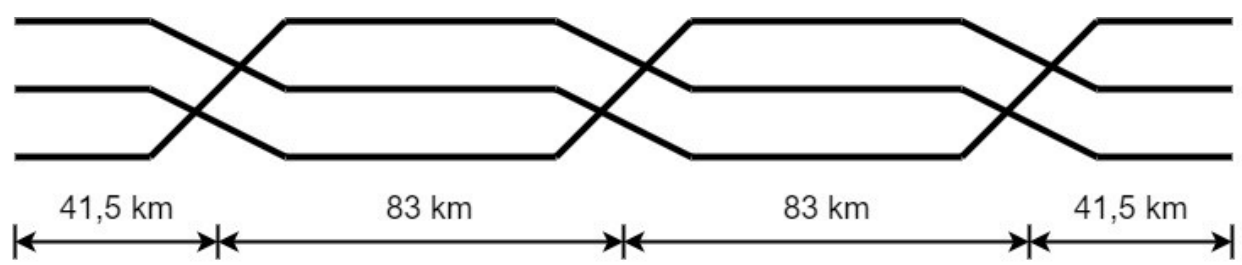

Figure 6. Complete transposition cycle.

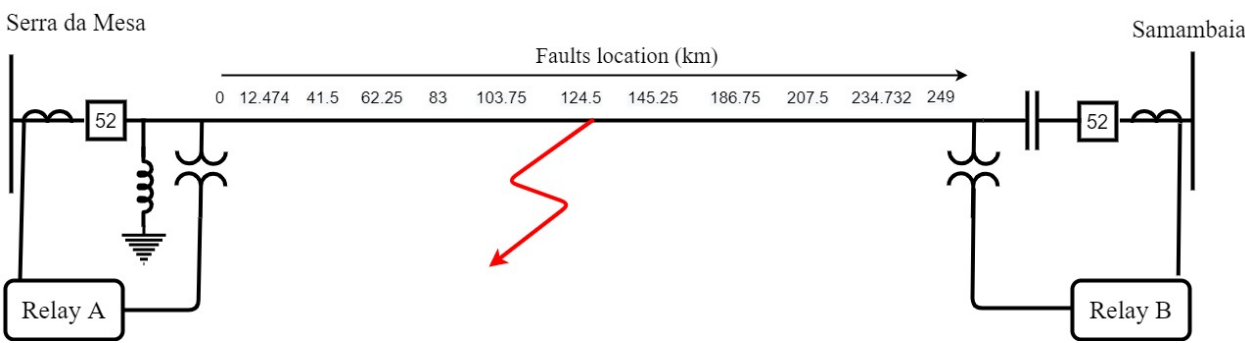

Figure 7. Single-line diagram of Serra da Mesa - Samambaia C3 transmission line.

\begin{tabular}{lc}
\hline \multicolumn{2}{c}{ Simulated fault types } \\
\hline Phase-ground & AG, BG, CG \\
Phase-phase-ground & ABG, ACG, BCG \\
Three-phase & ABC \\
Phase-phase & AB, AC, BC \\
Three-phase-ground & ABCG \\
\hline
\end{tabular}

TABLE 3. Fault types. 


\begin{tabular}{lllr}
\hline \multicolumn{2}{c}{ Positive sequence $(\Omega)$} & \multicolumn{2}{c}{ Zero sequence $(\Omega)$} \\
\hline Primary & $66.83 \angle 86.54^{\circ}$ & Primary & $273.82 \angle 71.29^{\circ}$ \\
Secondary & $44.55 \angle 86.54^{\circ}$ & Secondary & $182.55 \angle 71.29^{\circ}$ \\
\hline
\end{tabular}

TABLE 4. Impedance data.

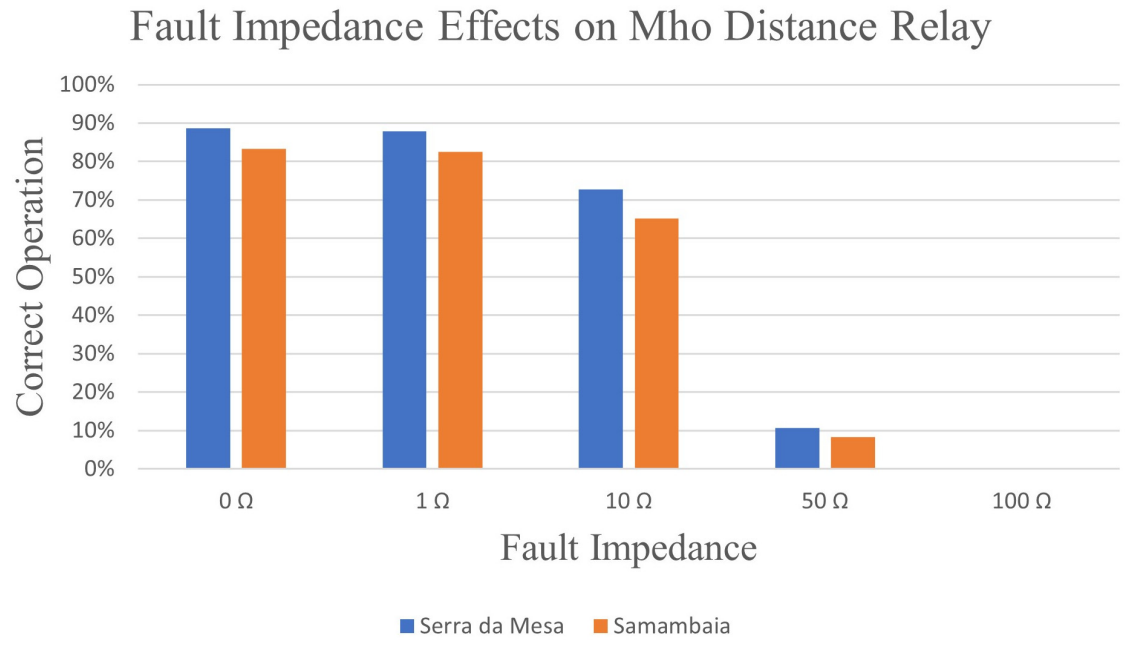

FiguRE 8. Correct operation of the distance relay considering effects of fault resistance.

relay settings and to the calculation of the zero-sequence compensation factor $k$ in 8 . Table 4 presents these impedance values.

The zero-sequence compensation factor in 8 and the reach settings denoted in Table 1 are calculated using the secondary impedances. These secondary impedances are calculated using the turns ratio regarding the voltage transformer and the current transformer. The voltage transformer turns ratio is denoted as VTR $=$ 4500 , the current transformer turns ratio as $\mathrm{CTR}=3000$. The calculation of the secondary impedances uses 16

$$
Z_{\text {secondary }}=Z_{\text {primary }} \times \frac{C T R}{V T R}
$$

The first generated result, presented in Figure 8 is a graph regarding the distance relay operation. It includes a comparison considering its correct operation facing different fault resistance values.

Analysing the results in Figure 8 it is possible to notice the derating of the distance relay's performance as the fault impedance increases. Even in the presence of faults with a low impedance, this distance relay does not operate with a $100 \%$ accuracy. Other observations include, for phase-ground faults close to the relay location, an unwanted encroachment of phase elements into the relay's operation zone might occur, resulting in unwanted trips. For faults near the remote end, the impedance path might dynamically enter and leave the protection zone 2 from time to time. Thus, its operation might not occur within the expected time. The effect of fault resistance can be better understood analysing Figure 9 The impedance path for a phase A - ground fault located in the middle of the transmission line's length, seen by the relay located at the Serra da Mesa substation is shown.

From Figures 8 and 9 it can be seen that for larger values of fault resistance, the impedance path calculated by the digital relay underreaches the protection zone.

Figure 10 details an unwanted path encroachment of a phase element for a phase-ground fault. A bolted fault between the phase B and the ground close to the relay's location at Serra da Mesa substation was simulated, and there was an incorrect operation condition. As it is shown in Figure 10, there was an unwanted encroachment of the phase element $Z_{b c}$ and the distance relay sent trip signals to both phase $\mathrm{B}$ and phase $\mathrm{C}$ circuit-breakers. This was not supposed to happen.

The last case evaluated in this paper is the confidence interval calculation for the fault detection time. This evaluation considers several different simulated scenarios. Statements defined in [29] are used for the confidence interval calculation. So, it is possible to provide an interval of plausible values for the fault detection time and demonstrate how confident they are. The fault detection time for the protection zone 1 and protection zone 2 was calculated, considering all the simulated cases with the different fault types, fault locations, and fault 


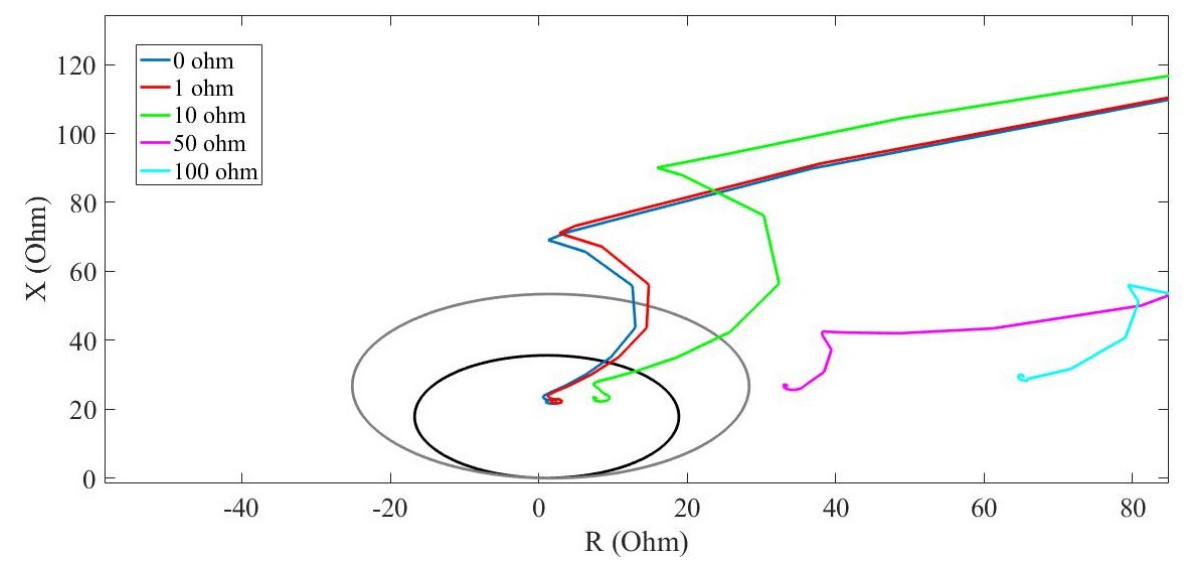

FiguRE 9. Effect of fault resistance on mho distance relay.

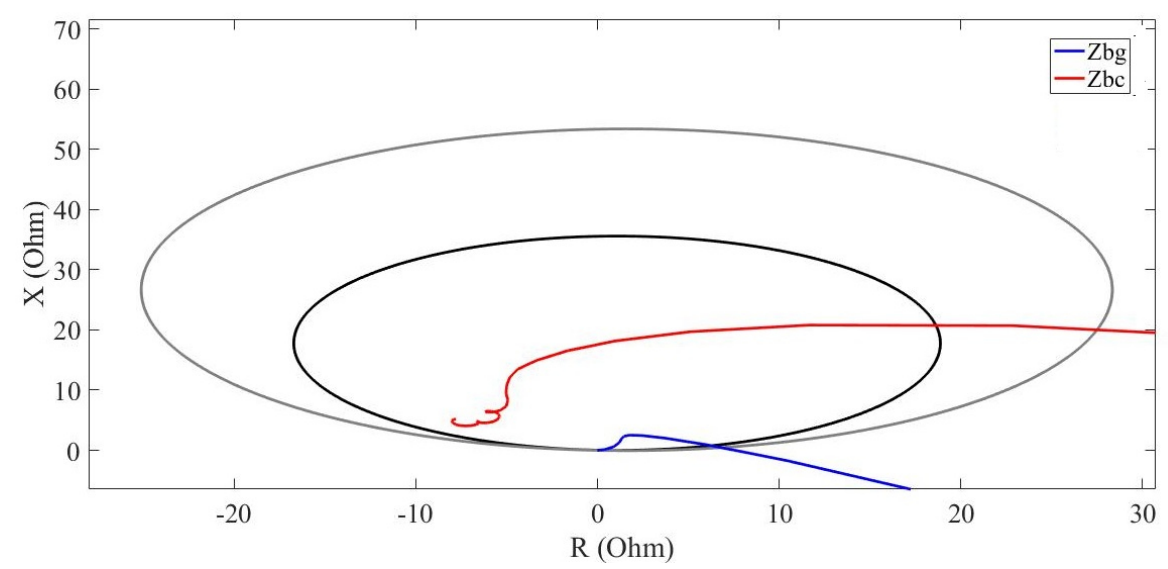

FiguRE 10. Unwanted encroachment of the $Z_{b c}$ element.

impedances. The confidence interval calculation only includes the cases with a correct operation by the distance relay. The result was a $95 \%$ confidence interval for the mean using 17 .

$$
\left(\bar{x}-1.96 \frac{\sigma}{\sqrt{n}}, \bar{x}+1.96 \frac{\sigma}{\sqrt{n}}\right)
$$

where:

$\bar{x}$ - mean time of fault detection

$\sigma$ - standard deviation of fault detection time

$n$ - number of samples

Table 5 shows the data required for the confidence interval computation. Figure 11 presents a graph for the confidence interval for the relays located at both substations, Serra da Mesa and Samambaia. Analysing Table 5 and Figure 11 it is possible to conclude that the fault detection time must not be treated as a constant parameter, as it usually is in the literature. It must be treated as a variable with a mean value and standard deviation subjected to many different conditions in electrical power systems. The fault detection time's upper and lower limits depend on what fault condition the relay is facing. Both the faults farther from the relay location and the faults with an impedance lead to a slower detection time. With the confidence interval computation, it is possible to have more details about the fault detection time and critical conditions for protection systems. Also, it is possible to determine if the protection systems comply to the requirements defined by power utilities, standards, and regulatory organizations. After these results, section 5 presents the conclusions.

\section{Conclusions}

This paper presented a detailed methodology on how to model a digital distance relay using PSCAD/EMTDC. The main aspects of a digital mho distance relay, its limitations, and how it can be used to protect transmission lines were shown. The basic steps used in its modelling include: 


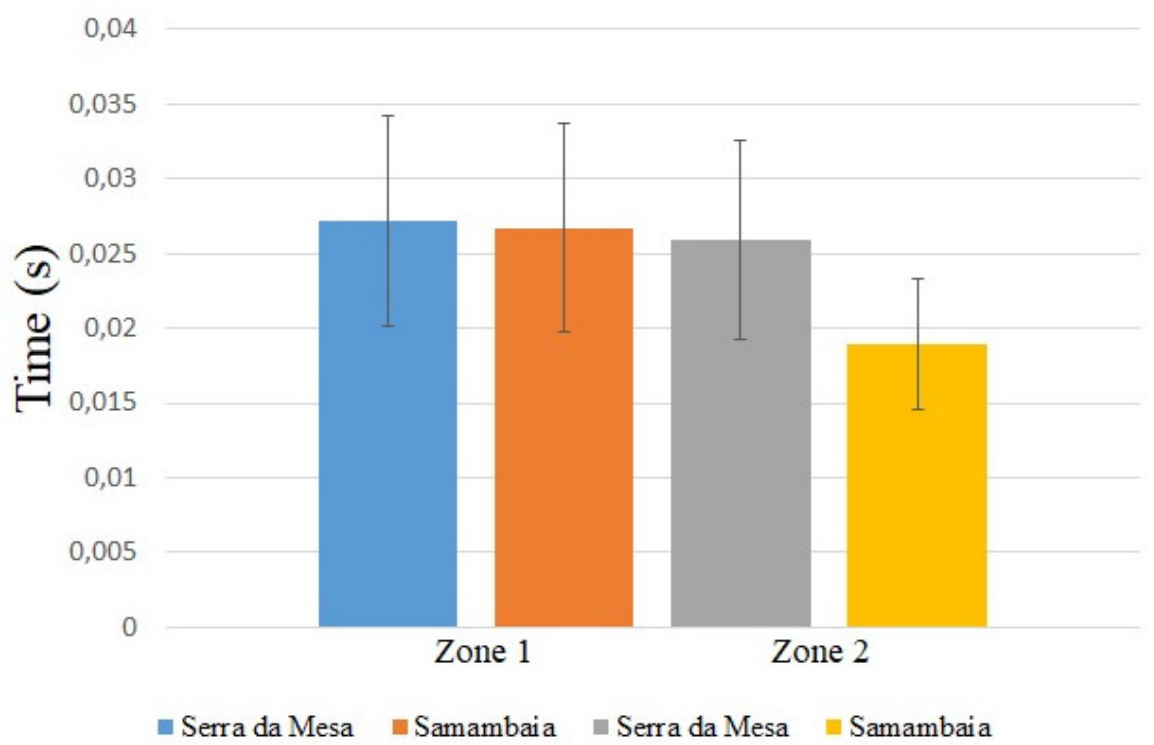

Figure 11. Confidence interval of fault detection time

\begin{tabular}{lccc}
\hline \multicolumn{4}{c}{ Zone 1} \\
\hline Substation & $\bar{x}(\mathrm{~s})$ & $\sigma(\mathrm{s})$ & $n$ \\
Serra da Mesa & 0.0272 & 0.0727 & 415 \\
Samambaia & 0.0267 & 0.0708 & 386 \\
\hline \multicolumn{4}{c}{ Zone 2} \\
\hline Substation & $\bar{x}(\mathrm{~s})$ & $\sigma(\mathrm{s})$ & $n$ \\
Serra da Mesa & 0.0259 & 0.0688 & 409 \\
Samambaia & 0.0189 & 0.044 & 380 \\
\hline
\end{tabular}

TABLE 5. Data to calculate the confidence interval of fault detection time.

- Signal conditioning unit

- Impedance calculation

- Fault detection

- Trip logical scheme

- Plotting the circular characteristic and impedance path

In the signal conditioning unit, the anti-aliasing filter was used to avoid noise from harmonics of higher frequencies. The data sampling, buffering, and the fast Fourier transform are implemented to estimate the voltage and current phasors in the fundamental frequency and remove any DC offset.

The impedance calculations for phase and ground elements are done using 6 6 . The flowchart for the trip decision is detailed in Figure 2, and the logical scheme to send trip signals to the circuit-breakers is shown in Figure 3. The plotting of the mho characteristic is demonstrated in a simplified and easy-to-understand manner through 12,15 The result is shown in Figure 4

Section 4 presents a detailed evaluation of the self-polarized mho distance relay. It is possible to notice that its incorrect operation is caused mainly by fault resistance effects, faults close to the relay's location, and faults close to the remote end.

A new approach on how to treat the fault detection time of digital distance relays is presented. In Figure 11 it is depicted that the fault detection time is not constant for every possible fault that might happen in electrical power systems. It is shown that the fault detection time varies within a time interval. This paper calculates a $95 \%$ confidence interval for the mean, showing upper and lower boundaries for this time interval.

The main scientific contributions of this paper are the modelling of a digital mho distance relay using PSCAD/EMTDC and the detailed evaluation of this relay in the following aspects:

- Effects of fault resistance on distance protection 
- Confidence interval calculation for the fault detection time

- Considerations about undesired conditions that might happen in distance protection.

\section{ACKNOWLEDGEMENTS}

The authors gratefully acknowledge the financial support by CAPES - Coordenação de Aperfeiçoamento de Pessoal de Nível Superior - Brasil and TAESA (R\&D) ANEEL Project PD - 07130-0053/2018.

\section{REFERENCES}

[1] S. H. Horowitz, A. G. Phadke. Power System Relaying, chap. Nonpilot Distance Protection of Transmission Lines, pp. 101-131. 3rd ed. John Wiley \& Sons, Ltd., 2008.

[2] C. A. B. Da Costa, N. S. D. Brito, B. A. De Souza. A methodology for distance relay modeling. IEEE Latin America Transactions 16(5):1388-1394, 2018. https://doi.org/10.1109/TLA.2018.8408432.

[3] R. H. Salim, D. P. Marzec, A. S. Bretas. Phase distance relaying with fault resistance compensation for unbalanced systems. IEEE Transactions on Power Delivery 26(2):1282-1283, 2011. https://doi.org/10.1109/TPWRD.2010.2052967

[4] J. Choi, Y. Han, I. Suh, et al. A study on the effects of new facts installations on existing distance protection relays. IFAC Proceedings Volumes 36(20):279-283, 2003. https://doi.org/10.1016/S1474-6670(17)34480-4.

[5] S. M. Hashemi, M. Sanaye-Pasand. Distance protection during asymmetrical power swings: Challenges and solutions. IEEE Transactions on Power Delivery 33(6):2736-2745, 2018. https://doi.org/10.1109/TPWRD.2018.2816304.

[6] A. Manori, M. Tripathy. Protection of TCSC transmission line by wavelet based advance Mho relay. In 2018 5th IEEE Uttar Pradesh Section International Conference on Electrical, Electronics and Computer Engineering (UPCON), pp. 1-4. 2018. https://doi.org/10.1109/UPCON.2018.8596759.

[7] P. R. Khade, M. P. Thakre. Optimal reach settings of Mho relay for series compensated transmission line protection. In 2020 4th International Conference on Electronics, Communication and Aerospace Technology (ICECA), p. 307-313. 2020. https://doi.org/10.1109/ICECA49313.2020.9297516

[8] Y. Liang, W. Li, W. Zha. Adaptive mho characteristic-based distance protection for lines emanating from photovoltaic power plants under unbalanced faults. IEEE Systems Journal pp. 1-11, 2020. https://doi.org/10.1109/JSYST.2020.3015225

[9] C. R. Mason. The art and science of protective relaying. Wiley, New York, 1956.

[10] H. Beleed, B. K. Johnson, H. L. Hess. An examination of the impact of D-FACTS on the dynamic behavior of mho and quadrilateral ground distance elements. In 2020 IEEE Power 8 Energy Society Innovative Smart Grid Technologies Conference (ISGT), pp. 1-5. 2020. https://doi.org/10.1109/ISGT45199.2020.9087712.

[11] L. Gérin-Lajoie. A MHO distance relay device in EMTPWorks. Electric Power Systems Research 79(3):484-491, 2009. https://doi.org/10.1016/j.epsr.2008.09.010

[12] K. Pipaliya, V. Makwana. Modeling and simulation of digital distance protection scheme for quadrilateral characteristics using PSCAD. International Journal of Advance Engineering and Research Development 2(5):1238-1246, 2015. https://doi.org/10.21090/ijaerd.0205173

[13] S. R. Samantaray, P. K. Dash. Transmission line distance relaying using a variable window short-time Fourier transform. Electric Power Systems Research 78(4):595-604, 2008. https://doi.org/10.1016/j.epsr.2007.05.005

[14] Manitoba HVDC Research Centre. Applications of PSCAD $D^{T M} / E M T D C^{T M}$. Winnipeg, 2008.

[15] Institute of Electrical and Electronic Engineers. Guide for protective relay applications to transmission lines. In IEEE Std C3\%.113-2015 (Revision of IEEE Std C37.113-1999), pp. 1-141. 2016.

[16] A. M. Abdullah, K. Butler-Purry. Distance protection zone 3 misoperation during system wide cascading events: The problem and a survey of solutions. Electric Power Systems Research 154:151-159, 2018. https://doi.org/10.1016/j.epsr.2017.08.023

[17] S. G. Aquiles Perez, M. S. Sachdev, T. S. Sidhu. Modeling relays for use in power system protection studies. In Canadian Conference on Electrical and Computer Engineering, 2005, pp. 566-569. 2005. https://doi.org/10.1109/CCECE.2005.1556994

[18] A. V. Oppenheim, R. W. Schafer. Discrete-Time Signal Processing, chap. Digital Processing of Analog Signals, pp. 153-237. 3rd ed. Pearson, 2010.

[19] A. M. Goler. PSCAD On-Line Help System, chap. PFFT 49 Low Pass, Anti-Aliasing Filter. PSCAD, Winnipeg, 1993.

[20] W. Kester. MT-002 TUTORIAL what the nyquist criterion means to your sampled data system design. Analog Devices pp. 1-12, 2009.

[21] R. Jayasinghe, S. Woodford. PSCAD On-Line Help System, chap. Online Fast Fourier Transform. PSCAD, Winnipeg, 1992. 
[22] M. Inci, M. Buyuk, M. Tumay. FFT based reference signal generation to compensate simultaneous voltage sag/swell and voltage harmonics. In 2016 IEEE 16th International Conference on Environment and Electrical Engineering (EEEIC), pp. 3-7. 2016. https://doi.org/10.1109/EEEIC.2016.7555876.

[23] K. R. Rao, D. N. Kim, J.-J. Hwang. Fast Fourier Transform - Algorithms and Applications. Springer Netherlands, Dordrecht, 2010.

[24] A. G. Phadke, J. S. Thorp. Computer Relaying for Power Systems, chap. Relaying Practices. Wiley, Chichester, UK, 2009.

[25] D. D. Fentie. Understanding the dynamic mho distance characteristic. In 2016 69th Annual Conference for Protective Relay Engineers (CPRE), pp. 1-15. 2016. https://doi.org/10.1109/CPRE.2016.7914922

[26] M. da C. Siqueira. Desempenho da proteção de distância sob diferentes formas de polarização, 2007. Federal University of Rio de Janeiro.

[27] B. Gustavsen, G. Irwin, R. Mangelrød, et al. International Converence on Power System Transients (IPST), chap. Transmission line models for the simulation of interaction phenomena between parallel AC and DC overhead lines, pp. 61-67. 1999.

[28] A. Morched, B. Gustavsen, M. Tartibi. A universal model for accurate calculation of electromagnetic transients on overhead lines and underground cables. IEEE Transactions on Power Delivery 14(3):1032-1038, 1999. https://doi.org/10.1109/61.772350

[29] F. M. Dekking, C. Kraaikamp, H. P. Lopuhaa, L. E. Meester. A Modern Introduction to Probability and Statistics, Understanding Why and How. Springer, London, 2005. 